\title{
A Series of New Copper Iodobismuthates: Structural Relationships, Optical Band Gaps Affected by Dimensionality, and Distinct Thermal Stabilities
}

\author{
Wen-Xiang Chai, ${ }^{\dagger, *}$ Li-Ming Wu, Jun-Qian Li, ${ }^{\S}$ Ling Chen ${ }^{*}{ }^{\dagger}$
}

State Key Laboratory of Structural Chemistry, Fujian Institute of Research on the Structure of Matter, Chinese Academy of Sciences, Fuzhou, Fujian 350002, Graduate School of the Chinese Academy of Sciences, Beijing 100039, and Department of Chemistry, Fuzhou University, Fuzhou, Fujian 350002, P. R. China

\section{Supporting Figures:}

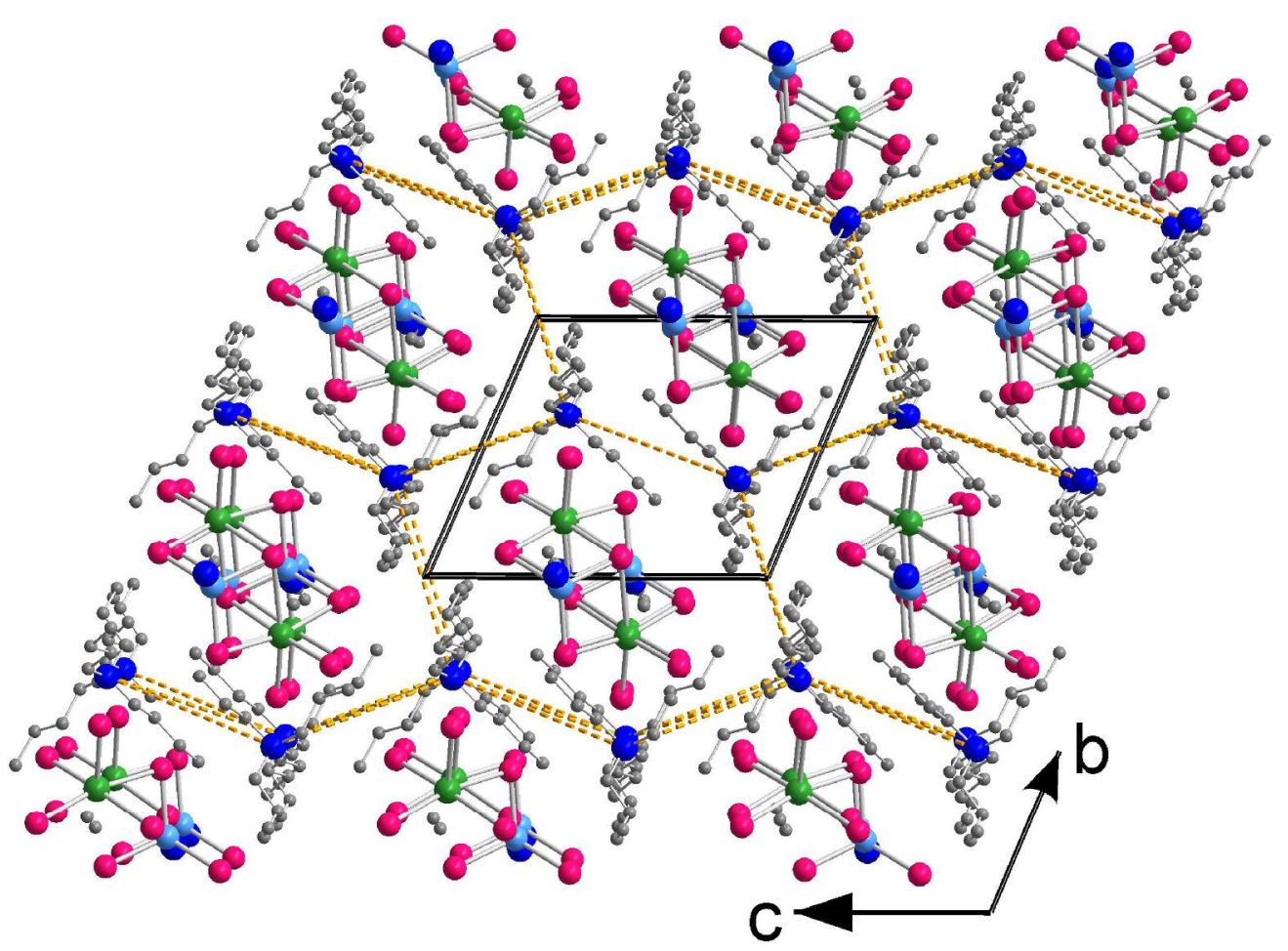

Figure S1. The packing view of 1 along $a$ axis: visual honeycomb-like channel of cation arrays and centric anions. Bi: green; $\mathrm{Cu}$ : blue; I: red; N: deep blue; C: gray, and hydrogen atoms are omitted for clarity. 


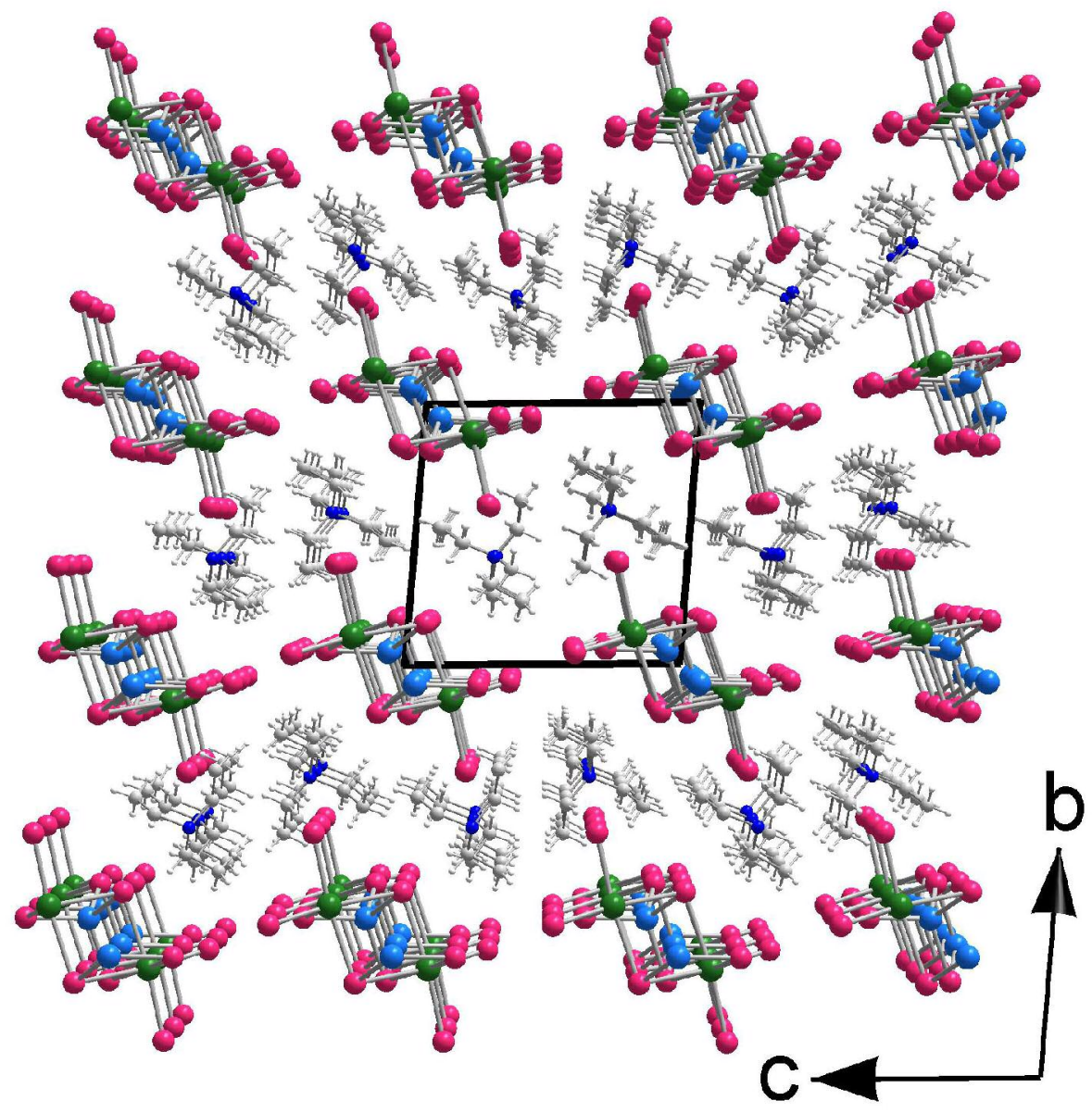

Figure S2. The packing view of samdwich type structure in $\mathbf{2}$ looking down a axis. Green, red, blue, deep blue, pale gray and white balls present Bi, I, Ag, N, C and H atoms, respectively.

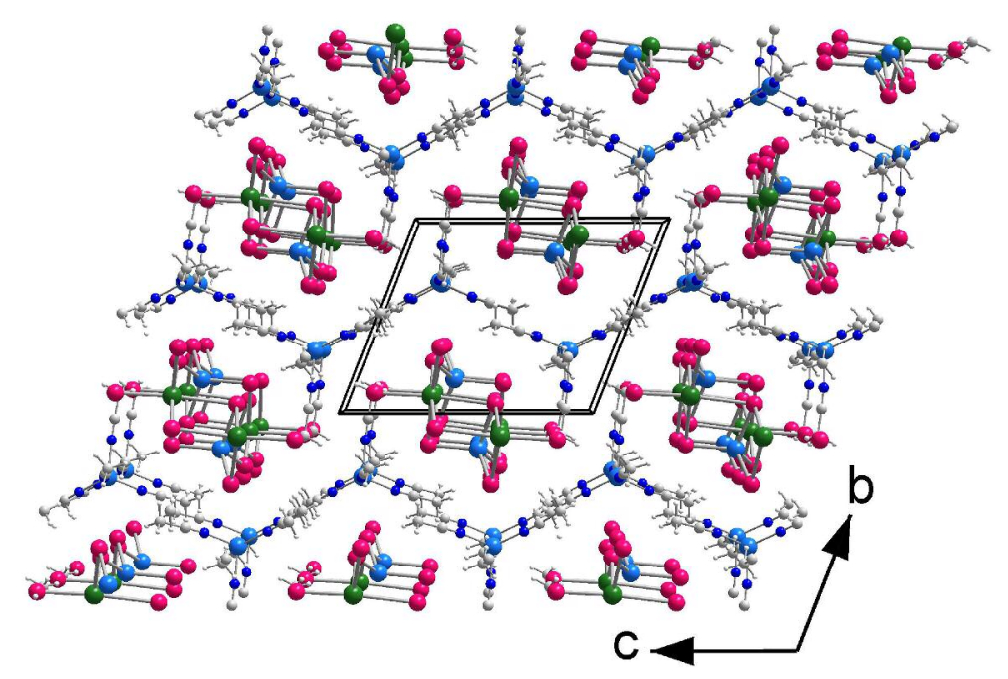

Figure S3. The packing view of $\mathbf{3}$ along $a$ axis: visual honeycomb-like channel of cation arrays and centric anions. Bi: green; $\mathrm{Cu}$ : blue; I: red; N: deep blue; C: gray; $\mathrm{H}$ : white. 
Figure S4. The Fourier Transform Infrared (FTIR) spectra of all compounds:

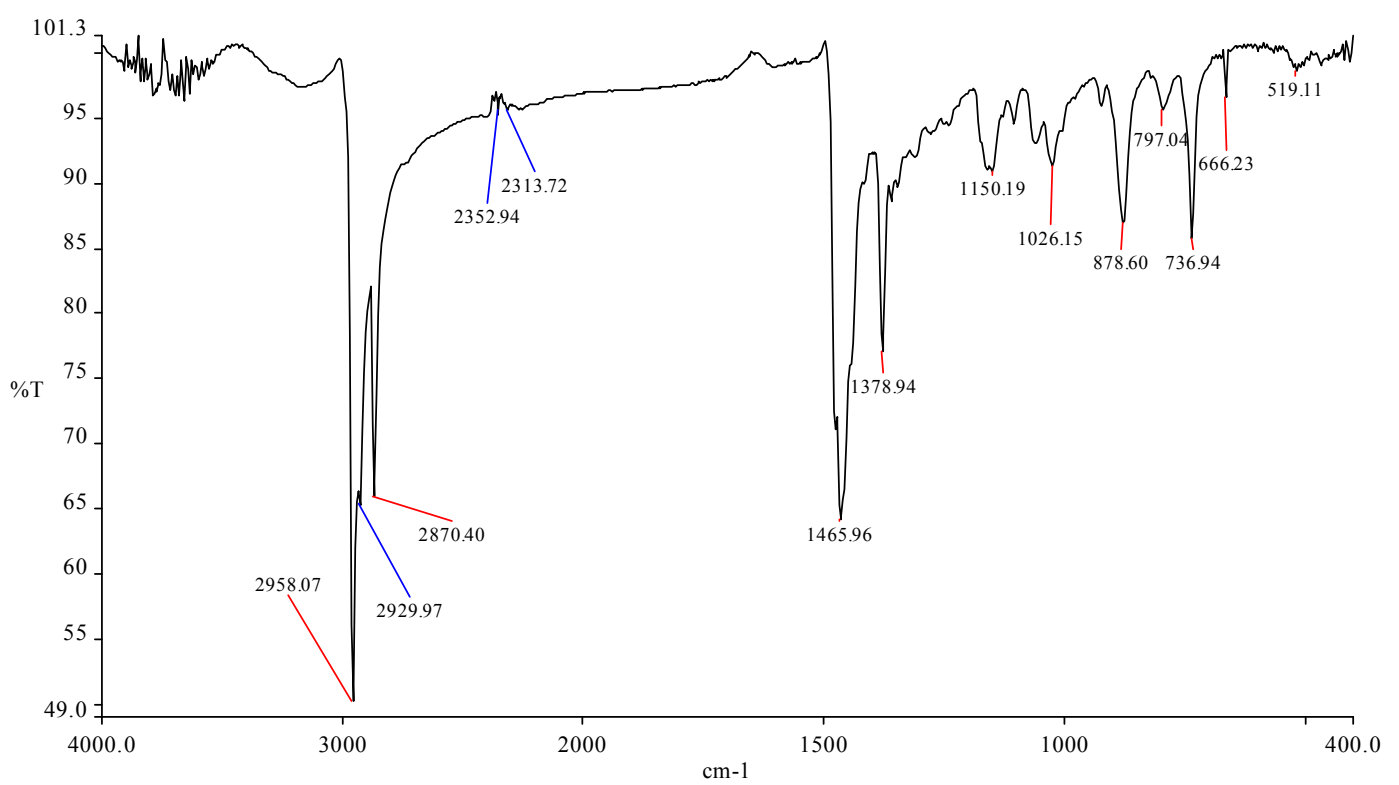

Figure S4-1. The IR spectrum of compound 1.

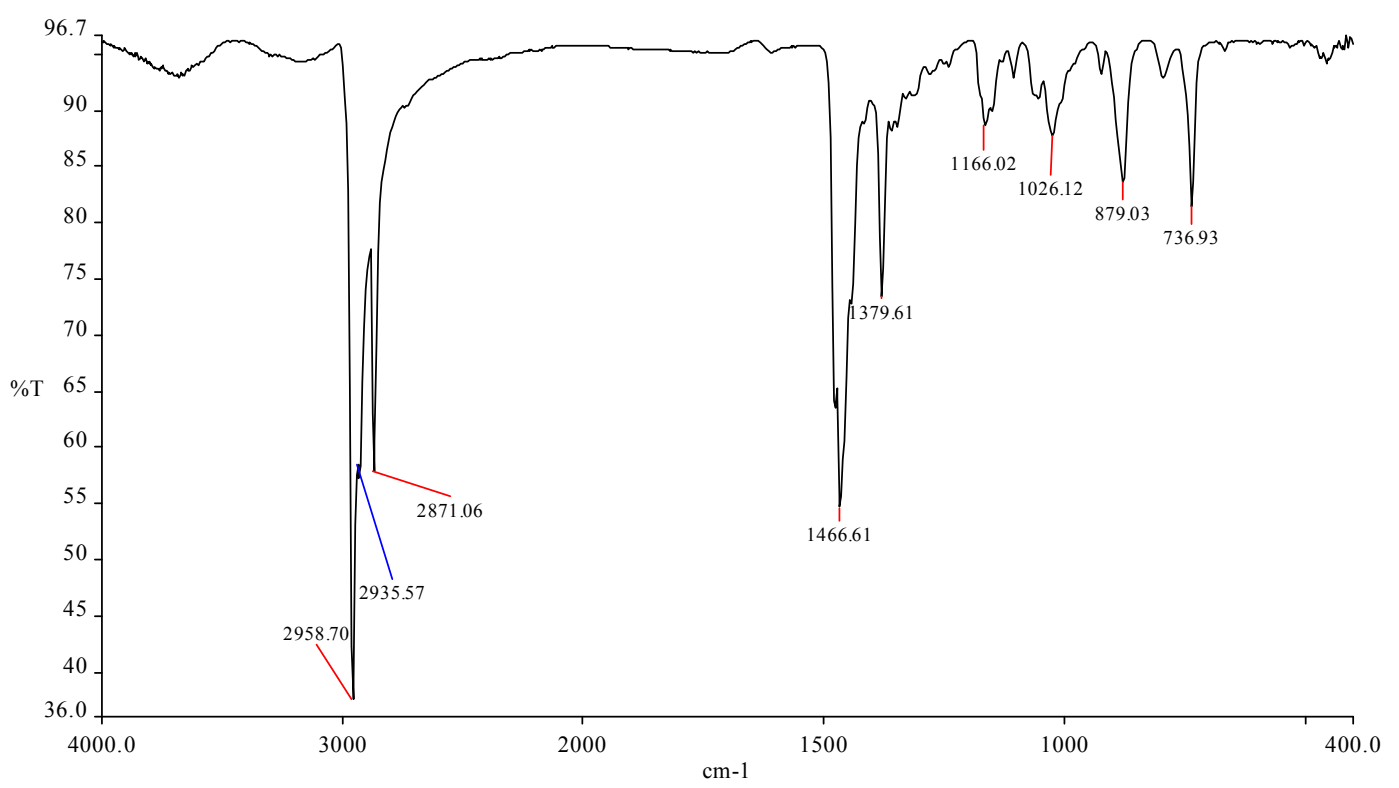

Figure S4-2. The IR spectrum of compound 1-A that is made by heating 1 at 100 under $\mathrm{N}_{2}$ atmosphere for 24 hours.

\begin{tabular}{|c|c|c|c|c|}
\hline & vibrations & frequency/ $\mathbf{c m}^{-1}$ & vibrations & frequency/ $\mathbf{c m}^{-1}$ \\
\hline \multirow{3}{*}{ Compound 1 } & $\mathbf{v}_{\mathrm{as}} \mathrm{CH}_{3}$ & 2958 & $\mathbf{v}_{\mathrm{as}} \mathrm{CH}_{2}$ & 2930 \\
\cline { 2 - 5 } & $\mathbf{v}_{\mathrm{s}} \mathrm{CH}_{3}$ & 2870 & $\mathbf{v} \mathrm{CN}$ & 2347 \\
\cline { 2 - 5 } & $\delta_{\mathrm{as}} \mathrm{CH}_{3}$ & 1466 & $\delta_{\mathrm{s}} \mathrm{CH}_{3}$ & 1379 \\
\hline \multirow{3}{*}{ compound 1-A } & $\mathbf{v}_{\mathrm{as}} \mathrm{CH}_{3}$ & 2959 & $\mathbf{v}_{\mathrm{as}} \mathrm{CH}_{2}$ & 2935 \\
\cline { 2 - 5 } & $\mathbf{v}_{\mathrm{s}} \mathrm{CH}_{3}$ & 2871 & $\delta_{\mathrm{as}} \mathrm{CH}_{3}$ & 1467 \\
\cline { 2 - 5 } & $\delta_{\mathrm{s}} \mathrm{CH}_{3}$ & 1379 & & \\
\hline
\end{tabular}




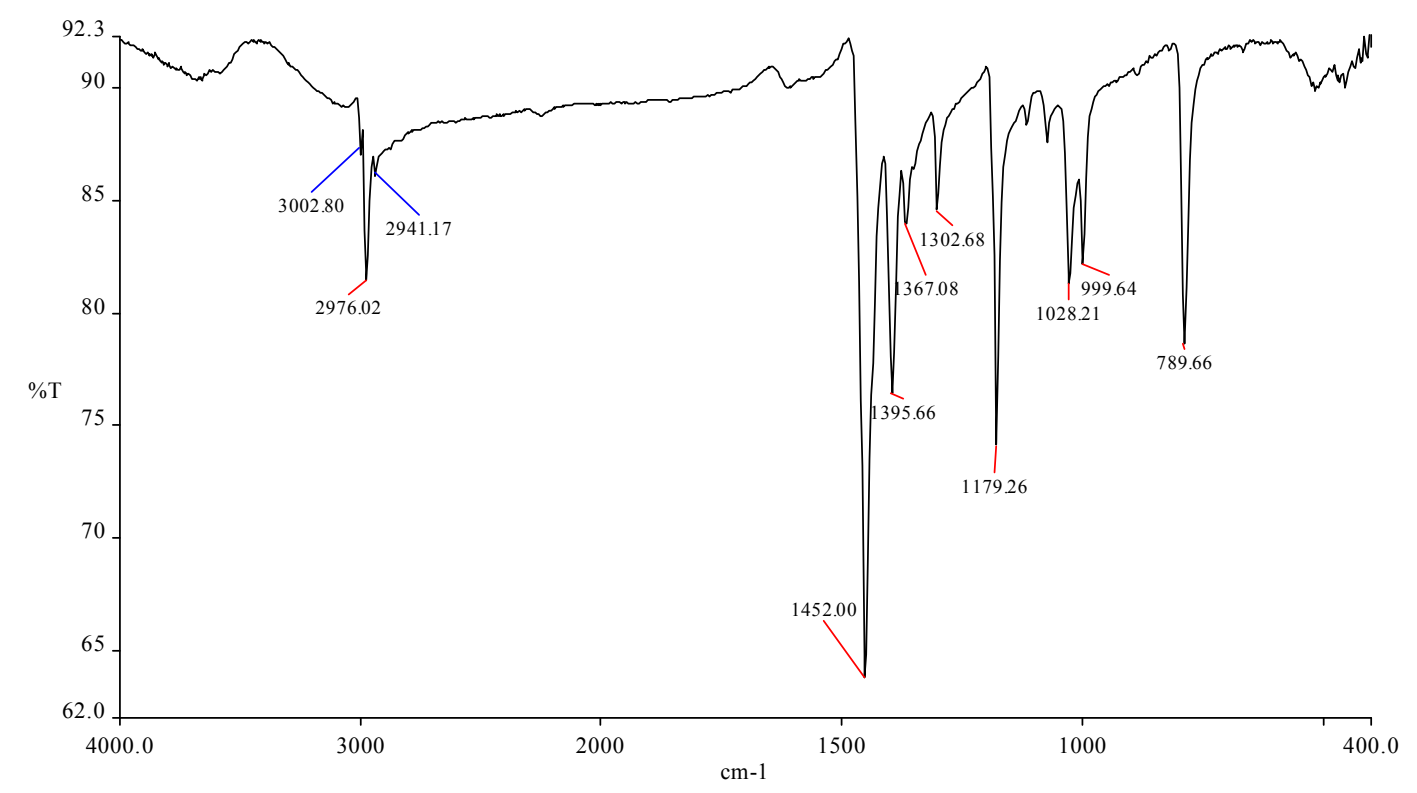

Figure S4-3. The IR spectrum of compound 2.

\begin{tabular}{|c|c|c|c|c|}
\hline & vibrations & frequency/ $\mathbf{c m}^{-1}$ & vibrations & frequency/ $\mathbf{c m}^{-1}$ \\
\hline \multirow{2}{*}{ Compound $\mathbf{2}$} & $\boldsymbol{v}_{\text {as }} \mathrm{CH}_{3}$ & 3003,2976 & $\boldsymbol{v}_{\text {as }} \mathrm{CH}_{2}$ & 2941 \\
\cline { 2 - 5 } & $\delta_{\text {as }} \mathrm{CH}_{3}$ & 1452 & $\delta_{\mathrm{s}} \mathrm{CH}_{3}$ & 1395 \\
\hline
\end{tabular}

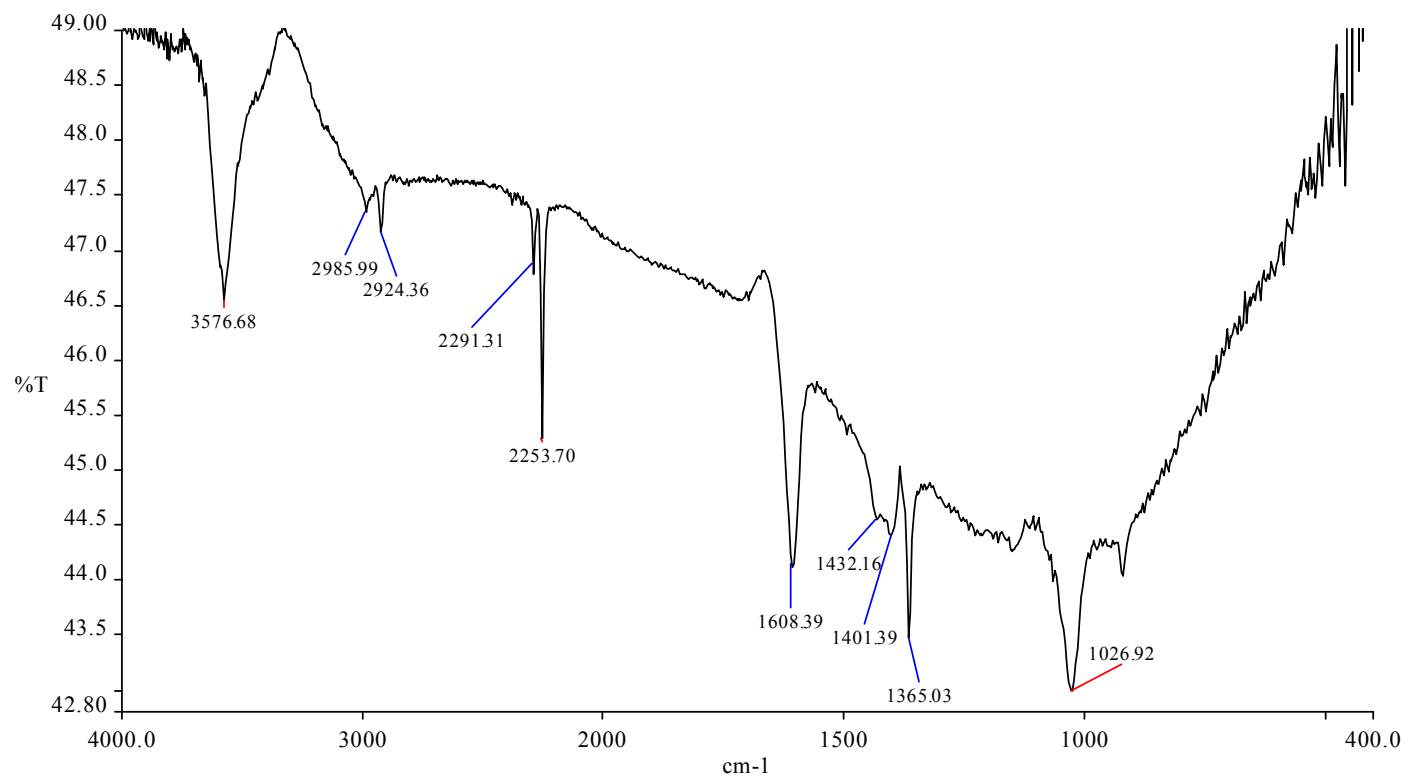

Figure S4-4. The IR spectrum of compound 3. 


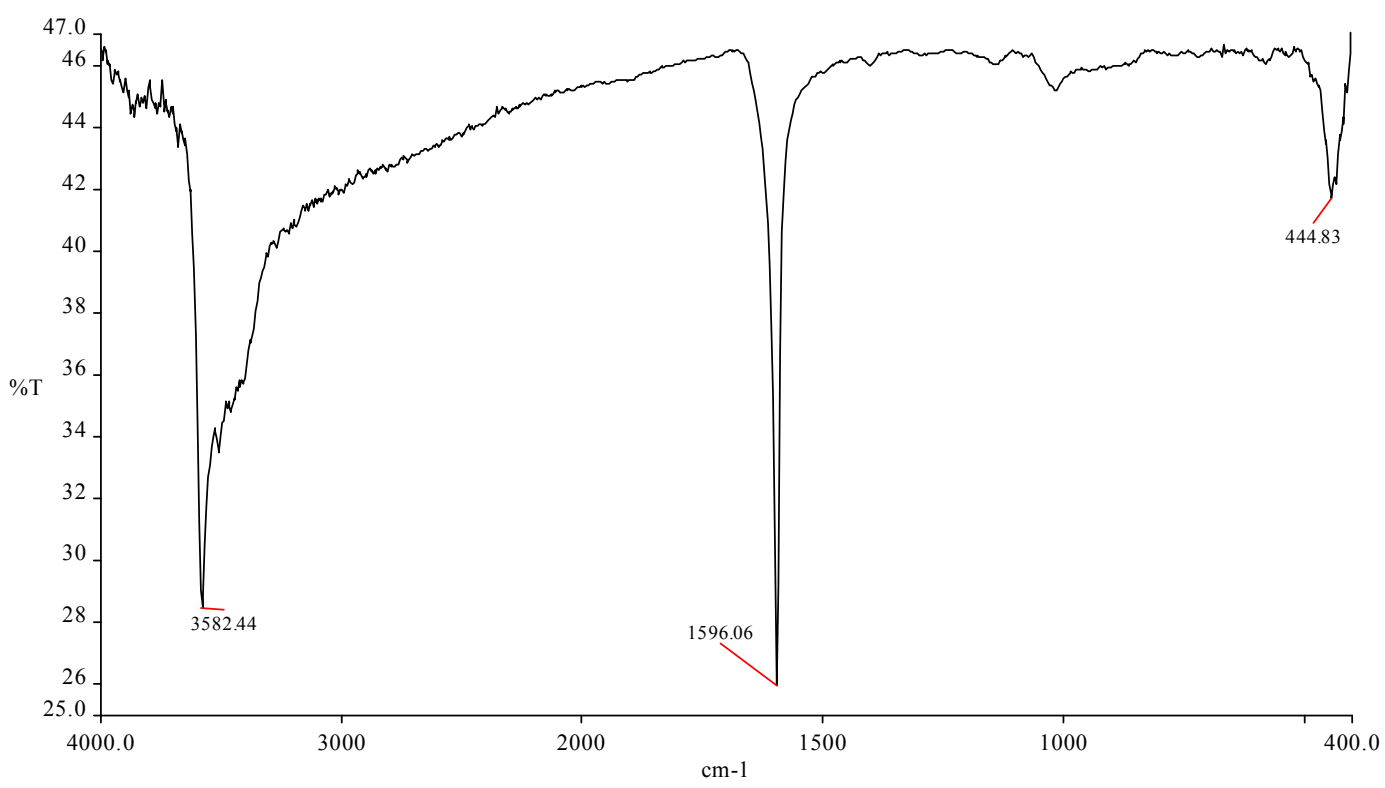

Figure S4-5. The IR spectrum of compound 3 heated at 100 under $\mathrm{N}_{2}$ atmosphere for 24 hours..

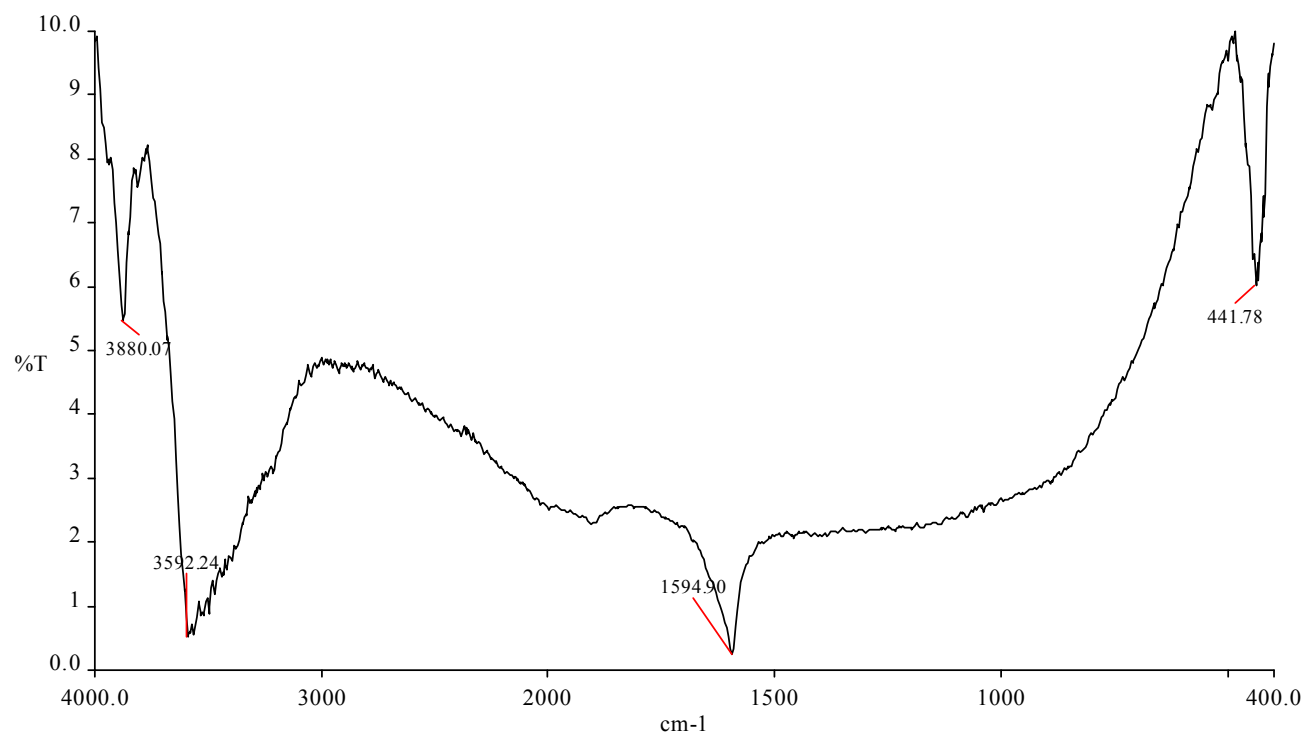

Figure S4-6. The IR spectrum of $\mathrm{BiI}_{3}$.

\begin{tabular}{|c|c|c|c|c|}
\hline & vibrations & frequency/ $\mathbf{c m}^{-1}$ & vibrations & frequency/ cm \\
\hline \multirow{4}{*}{ Compound 3} & & 3576 & & 1606 \\
\cline { 2 - 5 } & $\mathbf{v}_{\mathrm{as}} \mathrm{CH}_{3}$ & 2986 & $\mathbf{v}_{\mathrm{s}} \mathrm{CH}_{3}$ & 2924 \\
\cline { 2 - 5 } & $\mathbf{v C N}$ & 2286,2254 & $\delta_{\mathrm{as}} \mathrm{CH}_{3}$ & 1432,1404 \\
\cline { 2 - 5 } & $\delta_{\mathrm{s}} \mathrm{CH}_{3}$ & 1365 & & 1596 \\
\hline $\begin{array}{c}\text { compound } \mathbf{3} \\
\text { heated at } 100\end{array}$ & & 3582 & & 1595 \\
\hline $\mathrm{BiI}_{3}$ & & 3592 & & \\
\hline
\end{tabular}


2. Supporting Tables:

Table S1. Atomic coordinates and equivalent isotropic displacement parameters $\left(\AA^{2}\right)$ for compound $\mathbf{1}$.

\begin{tabular}{|l|l|l|l|l|}
\hline atom & $\mathbf{x}$ & $\mathbf{y}$ & $\mathbf{z}$ & $\mathbf{U}(\mathbf{e q})$ \\
\hline $\mathrm{Bi}(1)$ & $0.08379(2)$ & $0.21765(2)$ & $0.66323(2)$ & $0.04100(9)$ \\
\hline $\mathrm{I}(1)$ & $-0.01682(5)$ & $0.32604(5)$ & $0.84854(4)$ & $0.05844(14)$ \\
\hline $\mathrm{I}(2)$ & $0.33015(5)$ & $0.45635(6)$ & $0.72475(5)$ & $0.07693(18)$ \\
\hline $\mathrm{I}(3)$ & $0.17784(5)$ & $0.09365(5)$ & $0.78560(4)$ & $0.05666(14)$ \\
\hline $\mathrm{I}(4)$ & $-0.04638(5)$ & $0.28486(5)$ & $0.50789(4)$ & $0.05881(14)$ \\
\hline $\mathrm{I}(5)$ & $0.17552(4)$ & $0.07843(5)$ & $0.45184(4)$ & $0.04838(12)$ \\
\hline $\mathrm{Cu}(1)$ & $0.21014(10)$ & $-0.02809(12)$ & $0.58886(9)$ & $0.0713(3)$ \\
\hline $\mathrm{N}(1)$ & $0.3935(7)$ & $0.0180(8)$ & $0.6160(6)$ & $0.072(2)$ \\
\hline $\mathrm{N}(11)$ & $0.1776(5)$ & $0.3778(5)$ & $0.2107(5)$ & $0.0483(13)$ \\
\hline $\mathrm{C}(1)$ & $0.4956(8)$ & $0.0491(8)$ & $0.6386(7)$ & $0.063(2)$ \\
\hline $\mathrm{C}(2)$ & $0.6316(8)$ & $0.0938(10)$ & $0.6711(8)$ & $0.083(3)$ \\
\hline $\mathrm{C}(11)$ & $0.1755(8)$ & $0.2582(7)$ & $0.2216(7)$ & $0.060(2)$ \\
\hline $\mathrm{C}(12)$ & $0.1280(9)$ & $0.1388(8)$ & $0.1183(7)$ & $0.069(2)$ \\
\hline $\mathrm{C}(13)$ & $0.1796(11)$ & $0.0468(10)$ & $0.1241(8)$ & $0.085(3)$ \\
\hline $\mathrm{C}(14)$ & $0.3070(11)$ & $0.0841(12)$ & $0.1201(10)$ & $0.111(4)$ \\
\hline $\mathrm{C}(21)$ & $0.2077(8)$ & $0.4833(8)$ & $0.3264(6)$ & $0.066(2)$ \\
\hline $\mathrm{C}(22)$ & $0.3344(9)$ & $0.5357(10)$ & $0.3961(7)$ & $0.084(3)$ \\
\hline $\mathrm{C}(23)$ & $0.3501(10)$ & $0.6394(10)$ & $0.5049(8)$ & $0.090(3)$ \\
\hline $\mathrm{C}(24)$ & $0.4757(11)$ & $0.7033(12)$ & $0.5756(10)$ & $0.140(6)$ \\
\hline $\mathrm{C}(31)$ & $0.2776(7)$ & $0.4302(7)$ & $0.1456(7)$ & $0.0582(19)$ \\
\hline $\mathrm{C}(32)$ & $0.3056(8)$ & $0.5583(9)$ & $0.1384(8)$ & $0.072(2)$ \\
\hline $\mathrm{C}(33)$ & $0.3972(10)$ & $0.5913(10)$ & $0.0644(9)$ & $0.088(3)$ \\
\hline $\mathrm{C}(34)$ & $0.4308(10)$ & $0.7175(10)$ & $0.0516(9)$ & $0.096(3)$ \\
\hline $\mathrm{C}(41)$ & $0.0495(7)$ & $0.3443(8)$ & $0.1514(6)$ & $0.0551(18)$ \\
\hline $\mathrm{C}(42)$ & $-0.0632(8)$ & $0.2813(9)$ & $0.2009(7)$ & $0.067(2)$ \\
\hline $\mathrm{C}(43)$ & $-0.1861(10)$ & $0.2556(11)$ & $0.1396(9)$ & $0.089(3)$ \\
\hline $\mathrm{C}(44)$ & $-0.3032(9)$ & $0.1843(12)$ & $0.1791(11)$ & $0.105(4)$ \\
\hline $\mathrm{E}(1) \mathrm{fe})$ \\
\hline
\end{tabular}

$\mathrm{U}_{\text {eq }}$ is defined as one third of the trace of the orthogonalized $\mathrm{U}_{i j}$ tensor.

Table S2. Selected bond lengths $(\AA)$ and angles (deg.) for compound $\mathbf{1 .}$

\begin{tabular}{|l|l|}
\hline $\operatorname{Bi}(1)-\mathrm{I}(2)$ & $2.903(1)$ \\
\hline $\operatorname{Bi}(1)-\mathrm{I}(1)$ & $2.9448(9)$ \\
\hline $\operatorname{Bi}(1)-\mathrm{I}(3)$ & $3.0710(9)$ \\
\hline $\operatorname{Bi}(1)-\mathrm{I}(4)$ & $3.0940(9)$ \\
\hline $\operatorname{Bi}(1)-\mathrm{I}(5)$ & $3.2604(9)$ \\
\hline $\operatorname{Bi}(1)-\mathrm{I}(5) \# 1$ & $3.322(1)$ \\
\hline
\end{tabular}




\begin{tabular}{|l|l|}
\hline $\mathrm{Bi}(1)-\mathrm{Bi}(1) \# 1$ & $5.071(2)$ \\
\hline $\mathrm{I}(3)-\mathrm{Cu}(1)$ & $2.671(2)$ \\
\hline $\mathrm{I}(4)-\mathrm{Cu}(1) \# 1$ & $2.650(2)$ \\
\hline $\mathrm{I}(5)-\mathrm{Cu}(1)$ & $2.696(1)$ \\
\hline $\mathrm{I}(5)-\mathrm{Bi}(1) \# 1$ & $3.322(1)$ \\
\hline $\mathrm{Cu}(1)-\mathrm{N}(1)$ & $2.001(7)$ \\
\hline $\mathrm{Cu}(1)-\mathrm{I}(4) \# 1$ & $2.650(2)$ \\
\hline $\mathrm{N}(1)-\mathrm{C}(1)$ & $1.105(9)$ \\
\hline $\mathrm{N}(11)-\mathrm{C}(41)$ & $1.519(8)$ \\
\hline $\mathrm{N}(11)-\mathrm{C}(31)$ & $1.522(9)$ \\
\hline $\mathrm{N}(11)-\mathrm{C}(11)$ & $1.522(9)$ \\
\hline $\mathrm{N}(11)-\mathrm{C}(21)$ & $1.541(9)$ \\
\hline & \\
\hline $\mathrm{I}(2)-\mathrm{Bi}(1)-\mathrm{I}(1)$ & $95.10(3)$ \\
\hline $\mathrm{I}(2)-\mathrm{Bi}(1)-\mathrm{I}(3)$ & $93.87(3)$ \\
\hline $\mathrm{I}(1)-\mathrm{Bi}(1)-\mathrm{I}(3)$ & $93.04(3)$ \\
\hline $\mathrm{I}(2)-\mathrm{Bi}(1)-\mathrm{I}(4)$ & $95.83(3)$ \\
\hline $\mathrm{I}(1)-\mathrm{Bi}(1)-\mathrm{I}(4)$ & $92.13(3)$ \\
\hline $\mathrm{I}(3)-\mathrm{Bi}(1)-\mathrm{I}(4)$ & $168.56(2)$ \\
\hline $\mathrm{I}(2)-\mathrm{Bi}(1)-\mathrm{I}(5)$ & $88.97(3)$ \\
\hline $\mathrm{I}(1)-\mathrm{Bi}(1)-\mathrm{I}(5)$ & $175.93(2)$ \\
\hline $\mathrm{I}(3)-\mathrm{Bi}(1)-\mathrm{I}(5)$ & $86.87(3)$ \\
\hline $\mathrm{I}(4)-\mathrm{Bi}(1)-\mathrm{I}(5)$ & $87.26(3)$ \\
\hline $\mathrm{I}(2)-\mathrm{Bi}(1)-\mathrm{I}(5) \# 1$ & $168.18(2)$ \\
\hline $\mathrm{I}(1)-\mathrm{Bi}(1)-\mathrm{I}(5) \# 1$ & $96.70(3)$ \\
\hline $\mathrm{I}(3)-\mathrm{Bi}(1)-\mathrm{I}(5) \# 1$ & $86.26(3)$ \\
\hline $\mathrm{I}(4)-\mathrm{Bi}(1)-\mathrm{I}(5) \# 1$ & $83.01(3)$ \\
\hline $\mathrm{I}(5)-\mathrm{Bi}(1)-\mathrm{I}(5) \# 1$ & $79.23(3)$ \\
\hline $\mathrm{N}(1)-\mathrm{Cu}(1)-\mathrm{I}(4) \# 1$ & $114.4(2)$ \\
\hline $\mathrm{N}(1)-\mathrm{Cu}(1)-\mathrm{I}(3)$ & $103.9(2)$ \\
\hline $\mathrm{I}(4) \# 1-\mathrm{Cu}(1)-\mathrm{I}(3)$ & $115.27(5)$ \\
\hline $\mathrm{N}(1)-\mathrm{Cu}(1)-\mathrm{I}(5)$ & $109.1(2)$ \\
\hline $\mathrm{I}(4) \# 1-\mathrm{Cu}(1)-\mathrm{I}(5)$ & $105.48(5)$ \\
\hline $\mathrm{I}(3)-\mathrm{Cu}(1)-\mathrm{I}(5)$ & $108.48(5)$ \\
\hline $\mathrm{C}(41)-\mathrm{N}(11)-\mathrm{C}(31)$ & $108.2(5)$ \\
\hline $\mathrm{C}(41)-\mathrm{N}(11)-\mathrm{C}(11)$ & $112.3(6)$ \\
\hline $\mathrm{C}(31)-\mathrm{N}(11)-\mathrm{C}(11)$ & $109.0(5)$ \\
\hline $\mathrm{C}(41)-\mathrm{N}(11)-\mathrm{C}(21)$ & $107.3(6)$ \\
\hline $\mathrm{C}(31)-\mathrm{N}(11)-\mathrm{C}(21)$ & $111.3(6)$ \\
\hline $\mathrm{C}(11)-\mathrm{N}(11)-\mathrm{C}(21)$ & $108.8(6)$ \\
\hline
\end{tabular}

Symmetry transformations used to generate equivalent atoms:

$$
\text { \#1 -x,-y,-z+1 }
$$


Table S3. Atomic coordinates and equivalent isotropic displacement parameters $\left(\AA^{2}\right)$ for compound 2.

\begin{tabular}{|l|l|l|l|l|}
\hline atom & $\mathbf{x}$ & $\mathbf{y}$ & $\mathbf{z}$ & $\mathbf{U}(\mathbf{e q})$ \\
\hline $\mathrm{Bi}(1)$ & $0.63617(4)$ & $0.12073(3)$ & $0.18287(3)$ & $0.02929(12)$ \\
\hline $\mathrm{I}(1)$ & $0.68376(8)$ & $0.39147(6)$ & $0.25936(6)$ & $0.04521(18)$ \\
\hline $\mathrm{I}(2)$ & $0.84188(8)$ & $0.05836(7)$ & $0.38781(6)$ & $0.04894(19)$ \\
\hline $\mathrm{I}(3)$ & $0.32848(7)$ & $0.06638(6)$ & $0.27700(5)$ & $0.03800(16)$ \\
\hline $\mathrm{I}(4)$ & $0.92414(7)$ & $0.17781(5)$ & $0.03411(5)$ & $0.03409(15)$ \\
\hline $\mathrm{I}(5)$ & $0.61306(7)$ & $-0.16384(5)$ & $0.06896(5)$ & $0.03387(15)$ \\
\hline $\mathrm{Cu}(1)$ & $0.17522(16)$ & $0.05683(13)$ & $0.05621(12)$ & $0.0507(3)$ \\
\hline $\mathrm{N}(1)$ & $0.2217(8)$ & $0.5857(7)$ & $0.2996(6)$ & $0.0328(16)$ \\
\hline $\mathrm{C}(1)$ & $0.0912(11)$ & $0.6348(9)$ & $0.3609(8)$ & $0.039(2)$ \\
\hline $\mathrm{C}(2)$ & $0.0126(14)$ & $0.7344(11)$ & $0.3043(10)$ & $0.058(3)$ \\
\hline $\mathrm{C}(3)$ & $0.2836(11)$ & $0.4847(9)$ & $0.3714(8)$ & $0.041(2)$ \\
\hline $\mathrm{C}(4)$ & $0.1674(14)$ & $0.3737(11)$ & $0.3765(11)$ & $0.060(3)$ \\
\hline $\mathrm{C}(5)$ & $0.1417(12)$ & $0.5336(9)$ & $0.1744(8)$ & $0.044(2)$ \\
\hline $\mathrm{C}(6)$ & $0.2497(14)$ & $0.4766(12)$ & $0.0988(9)$ & $0.063(3)$ \\
\hline $\mathrm{C}(7)$ & $0.3568(11)$ & $0.6885(9)$ & $0.2915(8)$ & $0.044(2)$ \\
\hline $\mathrm{C}(8)$ & $0.4409(16)$ & $0.7542(12)$ & $0.4110(10)$ & $0.068(3)$ \\
\hline
\end{tabular}

$U_{\text {eq }}$ is defined as one third of the trace of the orthogonalized $U_{i j}$ tensor.

Table S4. Selected bond lengths ( $\AA$ ) and angles (deg.) for compound 2.

\begin{tabular}{|l|l|}
\hline $\mathrm{Bi}(1)-\mathrm{I}(2)$ & $2.8686(7)$ \\
\hline $\mathrm{Bi}(1)-\mathrm{I}(1)$ & $2.9689(7)$ \\
\hline $\mathrm{Bi}(1)-\mathrm{I}(3)$ & $2.9877(7)$ \\
\hline $\mathrm{Bi}(1)-\mathrm{I}(4)$ & $3.2152(7)$ \\
\hline $\mathrm{Bi}(1)-\mathrm{I}(5)$ & $3.2461(7)$ \\
\hline $\mathrm{Bi}(1)-\mathrm{I}(5) \# 1$ & $3.3899(7)$ \\
\hline $\mathrm{Bi}(1)-\mathrm{Bi}(1) \# 1$ & $4.8479(8)$ \\
\hline $\mathrm{I}(3)-\mathrm{Cu}(1)$ & $2.636(2)$ \\
\hline $\mathrm{I}(4)-\mathrm{Cu}(1) \# 2$ & $2.651(1)$ \\
\hline $\mathrm{I}(4)-\mathrm{Cu}(1) \# 1$ & $2.658(2)$ \\
\hline $\mathrm{I}(5)-\mathrm{Cu}(1) \# 1$ & $2.665(1)$ \\
\hline $\mathrm{I}(5)-\mathrm{Bi}(1) \# 1$ & $3.3899(7)$ \\
\hline $\mathrm{Cu}(1)-\mathrm{I}(4) \# 3$ & $2.651(1)$ \\
\hline $\mathrm{Cu}(1)-\mathrm{I}(4) \# 1$ & $2.658(2)$ \\
\hline $\mathrm{Cu}(1)-\mathrm{I}(5) \# 1$ & $2.665(1)$ \\
\hline $\mathrm{Cu}(1)-\mathrm{Cu}(1) \# 4$ & $3.100(3)$ \\
\hline $\mathrm{N}(1)-\mathrm{C}(7)$ & $1.51(1)$ \\
\hline $\mathrm{N}(1)-\mathrm{C}(3)$ & $1.52(1)$ \\
\hline
\end{tabular}




\begin{tabular}{|l|l|}
\hline $\mathrm{N}(1)-\mathrm{C}(5)$ & $1.52(1)$ \\
\hline $\mathrm{N}(1)-\mathrm{C}(1)$ & $1.54(1)$ \\
\hline $\mathrm{I}(2)-\mathrm{Bi}(1)-\mathrm{I}(1)$ & \\
\hline $\mathrm{I}(2)-\mathrm{Bi}(1)-\mathrm{I}(3)$ & $94.90(2)$ \\
\hline $\mathrm{I}(1)-\mathrm{Bi}(1)-\mathrm{I}(3)$ & $96.43(2)$ \\
\hline $\mathrm{I}(2)-\mathrm{Bi}(1)-\mathrm{I}(4)$ & $93.94(2)$ \\
\hline $\mathrm{I}(1)-\mathrm{Bi}(1)-\mathrm{I}(4)$ & $93.93(2)$ \\
\hline $\mathrm{I}(3)-\mathrm{Bi}(1)-\mathrm{I}(4)$ & $88.01(2)$ \\
\hline $\mathrm{I}(2)-\mathrm{Bi}(1)-\mathrm{I}(5)$ & $169.25(2)$ \\
\hline $\mathrm{I}(1)-\mathrm{Bi}(1)-\mathrm{I}(5)$ & $88.18(2)$ \\
\hline $\mathrm{I}(3)-\mathrm{Bi}(1)-\mathrm{I}(5)$ & $171.71(2)$ \\
\hline $\mathrm{I}(4)-\mathrm{Bi}(1)-\mathrm{I}(5)$ & $93.36(2)$ \\
\hline $\mathrm{I}(2)-\mathrm{Bi}(1)-\mathrm{I}(5) \# 1$ & $84.10(2)$ \\
\hline $\mathrm{I}(1)-\mathrm{Bi}(1)-\mathrm{I}(5) \# 1$ & $174.29(2)$ \\
\hline $\mathrm{I}(3)-\mathrm{Bi}(1)-\mathrm{I}(5) \# 1$ & $90.81(2)$ \\
\hline $\mathrm{I}(4)-\mathrm{Bi}(1)-\mathrm{I}(5) \# 1$ & $83.18(2)$ \\
\hline $\mathrm{I}(5)-\mathrm{Bi}(1)-\mathrm{I}(5) \# 1$ & $86.23(2)$ \\
\hline $\mathrm{I}(3)-\mathrm{Cu}(1)-\mathrm{I}(4) \# 3$ & $86.16(2)$ \\
\hline $\mathrm{I}(3)-\mathrm{Cu}(1)-\mathrm{I}(4) \# 1$ & $113.54(5)$ \\
\hline $\mathrm{I}(4) \# 3-\mathrm{Cu}(1)-\mathrm{I}(4) \# 1$ & $111.04(5)$ \\
\hline $\mathrm{I}(3)-\mathrm{Cu}(1)-\mathrm{I}(5) \# 1$ & $108.54(5)$ \\
\hline $\mathrm{I}(4) \# 3-\mathrm{Cu}(1)-\mathrm{I}(5) \# 1$ & $106.39(5)$ \\
\hline $\mathrm{I}(4) \# 1-\mathrm{Cu}(1)-\mathrm{I}(5) \# 1$ & $108.40(5)$ \\
\hline $\mathrm{C}(7)-\mathrm{N}(1)-\mathrm{C}(3)$ & $108.79(5)$ \\
\hline $\mathrm{C}(7)-\mathrm{N}(1)-\mathrm{C}(5)$ & $110.8(7)$ \\
\hline $\mathrm{C}(3)-\mathrm{N}(1)-\mathrm{C}(5)$ & $108.7(7)$ \\
\hline $\mathrm{C}(7)-\mathrm{N}(1)-\mathrm{C}(1)$ & $111.2(7)$ \\
\hline $\mathrm{C}(3)-\mathrm{N}(1)-\mathrm{C}(1)$ & $111.6(7)$ \\
\hline $\mathrm{C}(5)-\mathrm{N}(1)-\mathrm{C}(1)$ & $107.5(6)$ \\
\hline
\end{tabular}

Symmetry transformations used to generate equivalent atoms:

$$
\begin{aligned}
& \# 1-\mathrm{x}+1,-\mathrm{y},-\mathrm{z} \quad \# 2 \mathrm{x}+1, \mathrm{y}, \mathrm{z} \quad \# 3 \mathrm{x}-1, \mathrm{y}, \mathrm{z} \\
& \# 4-\mathrm{x},-\mathrm{y},-\mathrm{z}
\end{aligned}
$$

Table S5. Atomic coordinates and equivalent isotropic displacement parameters $\left(\AA^{2}\right)$ for compound 3.

\begin{tabular}{|l|l|l|l|l|}
\hline atom & $\mathbf{x}$ & $\mathbf{y}$ & $\mathbf{z}$ & $\mathbf{U}(\mathbf{e q})$ \\
\hline $\mathrm{Bi}(1)$ & $0.35096(7)$ & $0.09887(6)$ & $0.66230(5)$ & $0.0318(2)$ \\
\hline $\mathrm{I}(1)$ & $0.38129(18)$ & $0.11499(15)$ & $0.88526(10)$ & $0.0589(4)$ \\
\hline $\mathrm{I}(2)$ & $0.06104(14)$ & $0.26941(12)$ & $0.70915(10)$ & $0.0439(4)$ \\
\hline $\mathrm{I}(3)$ & $0.56990(14)$ & $0.34456(12)$ & $0.70115(10)$ & $0.0444(4)$ \\
\hline $\mathrm{I}(4)$ & $0.16561(15)$ & $-0.15370(12)$ & $0.59913(9)$ & $0.0440(3)$ \\
\hline
\end{tabular}




\begin{tabular}{|l|l|l|l|l|}
\hline $\mathrm{I}(5)$ & $0.31772(13)$ & $0.05683(11)$ & $0.40249(8)$ & $0.0344(3)$ \\
\hline $\mathrm{Cu}(1)$ & $0.7917(3)$ & $0.1747(3)$ & $0.60052(19)$ & $0.0543(7)$ \\
\hline $\mathrm{Cu}(2)$ & $0.0160(3)$ & $0.3252(3)$ & $0.1921(2)$ & $0.0573(7)$ \\
\hline $\mathrm{N}(1)$ & $0.084(2)$ & $0.1354(19)$ & $0.1434(13)$ & $0.062(5)$ \\
\hline $\mathrm{N}(2)$ & $0.106(2)$ & $0.3985(16)$ & $0.3405(13)$ & $0.054(4)$ \\
\hline $\mathrm{N}(3)$ & $0.117(2)$ & $0.4059(15)$ & $0.0946(13)$ & $0.051(4)$ \\
\hline $\mathrm{N}(4)$ & $-0.220(2)$ & $0.3189(16)$ & $0.1915(12)$ & $0.049(4)$ \\
\hline $\mathrm{C}(1)$ & $0.109(2)$ & $0.028(2)$ & $0.1216(14)$ & $0.046(5)$ \\
\hline $\mathrm{C}(2)$ & $0.142(3)$ & $-0.108(2)$ & $0.0968(18)$ & $0.080(7)$ \\
\hline $\mathrm{C}(3)$ & $0.175(3)$ & $0.428(2)$ & $0.4193(16)$ & $0.058(6)$ \\
\hline $\mathrm{C}(4)$ & $0.259(3)$ & $0.462(2)$ & $0.5223(17)$ & $0.074(7)$ \\
\hline $\mathrm{C}(5)$ & $0.184(2)$ & $0.450(2)$ & $0.0471(15)$ & $0.048(5)$ \\
\hline $\mathrm{C}(6)$ & $0.269(3)$ & $0.516(2)$ & $-0.0127(16)$ & $0.062(6)$ \\
\hline $\mathrm{C}(7)$ & $-0.351(3)$ & $0.2956(18)$ & $0.1982(13)$ & $0.047(5)$ \\
\hline $\mathrm{C}(8)$ & $-0.527(2)$ & $0.264(2)$ & $0.2102(18)$ & $0.066(6)$ \\
\hline
\end{tabular}

$\mathrm{U}_{\mathrm{eq}}$ is defined as one third of the trace of the orthogonalized $\mathrm{U}_{i j}$ tensor.

Table S6. Selected bond lengths ( $\AA$ ) and angles (deg.) for compound 3.

\begin{tabular}{|l|l|}
\hline $\mathrm{Bi}(1)-\mathrm{I}(1)$ & $2.923(2)$ \\
\hline $\mathrm{Bi}(1)-\mathrm{I}(2)$ & $3.000(2)$ \\
\hline $\mathrm{Bi}(1)-\mathrm{I}(4)$ & $3.003(2)$ \\
\hline $\mathrm{Bi}(1)-\mathrm{I}(3)$ & $3.129(2)$ \\
\hline $\mathrm{Bi}(1)-\mathrm{I}(5) \# 1$ & $3.218(2)$ \\
\hline $\mathrm{Bi}(1)-\mathrm{I}(5)$ & $3.334(2)$ \\
\hline $\mathrm{Bi}(1)-\mathrm{Bi}(1) \# 1$ & $4.803(2)$ \\
\hline $\mathrm{I}(2)-\mathrm{Cu}(1) \# 2$ & $2.652(3)$ \\
\hline $\mathrm{I}(3)-\mathrm{Cu}(1)$ & $2.643(3)$ \\
\hline $\mathrm{I}(4)-\mathrm{Cu}(1) \# 1$ & $2.616(3)$ \\
\hline $\mathrm{I}(4)-\mathrm{Cu}(1) \# 2$ & $4.786(3)$ \\
\hline $\mathrm{I}(5)-\mathrm{Cu}(1) \# 1$ & $2.685(3)$ \\
\hline $\mathrm{I}(5)-\mathrm{Bi}(1) \# 1$ & $3.218(2)$ \\
\hline $\mathrm{Cu}(1)-\mathrm{I}(4) \# 1$ & $2.616(3)$ \\
\hline $\mathrm{Cu}(1)-\mathrm{I}(2) \# 3$ & $2.652(3)$ \\
\hline $\mathrm{Cu}(1)-\mathrm{I}(5) \# 1$ & $2.685(3)$ \\
\hline $\mathrm{Cu}(1)-\mathrm{Cu}(1) \# 4$ & $5.185(5)$ \\
\hline $\mathrm{Cu}(2)-\mathrm{N}(4)$ & $1.97(2)$ \\
\hline $\mathrm{Cu}(2)-\mathrm{N}(2)$ & $1.99(2)$ \\
\hline $\mathrm{Cu}(2)-\mathrm{N}(3)$ & $2.01(2)$ \\
\hline $\mathrm{Cu}(2)-\mathrm{N}(1)$ & $2.04(2)$ \\
\hline $\mathrm{N}(1)-\mathrm{C}(1)$ & $1.13(2)$ \\
\hline $\mathrm{N}(2)-\mathrm{C}(3)$ & $1.13(2)$ \\
\hline $\mathrm{N}(3)-\mathrm{C}(5)$ & $1.08(2)$ \\
\hline & \\
\hline
\end{tabular}




\begin{tabular}{|l|l|}
\hline $\mathrm{N}(4)-\mathrm{C}(7)$ & $1.13(2)$ \\
\hline $\mathrm{I}(1)-\mathrm{Bi}(1)-\mathrm{I}(2)$ & $92.84(4)$ \\
\hline $\mathrm{I}(1)-\mathrm{Bi}(1)-\mathrm{I}(4)$ & $91.76(4)$ \\
\hline $\mathrm{I}(2)-\mathrm{Bi}(1)-\mathrm{I}(4)$ & $95.29(5)$ \\
\hline $\mathrm{I}(1)-\mathrm{Bi}(1)-\mathrm{I}(3)$ & $93.40(4)$ \\
\hline $\mathrm{I}(2)-\mathrm{Bi}(1)-\mathrm{I}(3)$ & $89.80(5)$ \\
\hline $\mathrm{I}(4)-\mathrm{Bi}(1)-\mathrm{I}(3)$ & $172.57(4)$ \\
\hline $\mathrm{I}(1)-\mathrm{Bi}(1)-\mathrm{I}(5) \# 1$ & $92.02(4)$ \\
\hline $\mathrm{I}(2)-\mathrm{Bi}(1)-\mathrm{I}(5) \# 1$ & $172.07(4)$ \\
\hline $\mathrm{I}(4)-\mathrm{Bi}(1)-\mathrm{I}(5) \# 1$ & $90.82(5)$ \\
\hline $\mathrm{I}(3)-\mathrm{Bi}(1)-\mathrm{I}(5) \# 1$ & $83.66(5)$ \\
\hline $\mathrm{I}(1)-\mathrm{Bi}(1)-\mathrm{I}(5)$ & $175.81(4)$ \\
\hline $\mathrm{I}(2)-\mathrm{Bi}(1)-\mathrm{I}(5)$ & $89.79(3)$ \\
\hline $\mathrm{I}(4)-\mathrm{Bi}(1)-\mathrm{I}(5)$ & $84.75(3)$ \\
\hline $\mathrm{I}(3)-\mathrm{Bi}(1)-\mathrm{I}(5)$ & $89.87(3)$ \\
\hline $\mathrm{I}(5) \# 1-\mathrm{Bi}(1)-\mathrm{I}(5)$ & $85.73(3)$ \\
\hline $\mathrm{I}(4) \# 1-\mathrm{Cu}(1)-\mathrm{I}(3)$ & $113.8(1)$ \\
\hline $\mathrm{I}(4) \# 1-\mathrm{Cu}(1)-\mathrm{I}(2) \# 3$ & $107.8(1)$ \\
\hline $\mathrm{I}(3)-\mathrm{Cu}(1)-\mathrm{I}(2) \# 3$ & $104.7(1)$ \\
\hline $\mathrm{I}(4) \# 1-\mathrm{Cu}(1)-\mathrm{I}(5) \# 1$ & $107.60(9)$ \\
\hline $\mathrm{I}(3)-\mathrm{Cu}(1)-\mathrm{I}(5) \# 1$ & $105.21(9)$ \\
\hline $\mathrm{I}(2) \# 3-\mathrm{Cu}(1)-\mathrm{I}(5) \# 1$ & $117.9(1)$ \\
\hline $\mathrm{N}(4)-\mathrm{Cu}(2)-\mathrm{N}(2)$ & $111.9(7)$ \\
\hline $\mathrm{N}(4)-\mathrm{Cu}(2)-\mathrm{N}(3)$ & $116.2(7)$ \\
\hline $\mathrm{N}(2)-\mathrm{Cu}(2)-\mathrm{N}(3)$ & $112.1(7)$ \\
\hline $\mathrm{N}(4)-\mathrm{Cu}(2)-\mathrm{N}(1)$ & $105.1(7)$ \\
\hline $\mathrm{N}(2)-\mathrm{Cu}(2)-\mathrm{N}(1)$ & $101.9(7)$ \\
\hline $\mathrm{N}(3)-\mathrm{Cu}(2)-\mathrm{N}(1)$ & $108.4(7)$ \\
\hline
\end{tabular}

Symmetry transformations used to generate equivalent atoms:

$$
\begin{aligned}
& \# 1-\mathrm{x}+1,-\mathrm{y},-\mathrm{z}+1 \quad \# 2 \mathrm{x}-1, \mathrm{y}, \mathrm{z} \quad \# 3 \mathrm{x}+1, \mathrm{y}, \mathrm{z} \\
& \# 4-\mathrm{x}+2,-\mathrm{y},-\mathrm{z}+1
\end{aligned}
$$

